\title{
Pacific
}

Journal of

Mathematics

\section{INTERPOLATION SUBMANIFOLDS OF THE UNITARY GROUP}

YEREN XU

Volume $165 \quad$ No. 1 


\title{
INTERPOLATION SUBMANIFOLDS OF THE UNITARY GROUP
}

\author{
YEREN XU
}

\begin{abstract}
An interpolation subset in the boundary of a domain is a closed set in which every continuous (or smooth) function can be extended as a holomorphic function inside the domain and continuous (or smooth, respectively) up to the boundary. In this paper we give some geometric description for submanifolds in the unitary group to be interpolation sets for the domain obtained by taking polynomial hull of the unitary group. In particular, we retrieved corresponding results on the polydisc.
\end{abstract}

The goal of this paper is to characterize the interpolation manifolds in the unitary group $\mathrm{U}(n)$, which is regarded as an $n^{2}$-dimensional real analytic submanifold in $C^{n^{2}}=R^{2 n^{2}}$. The theme of this topic started from the work by Henkin and Tumanov in [5], and Burns-Stout in [2] who proved the case for real analytic interpolation manifolds on the boundaries of some pseudoconvex domains. Then a lengthy cycle of works (Hakim-Sibony [4], Henkin-Tumanov [5], Stout [13], etc.) followed which mainly study the case for interpolation manifolds in the boundaries of strongly pseudoconvex domains. By using an embedding technique, Saeren's paper [11] was the first to deal with the case for the interpolation manifolds in the polydisc. In what follows, we shall deal with similar problems for $\mathrm{U}(n)$, which contains the polydisc as an $n$-dimensional real analytic submanifold.

The paper is organized in the following way.

In $\S 1$, we give some basic definitions and properties related to the unitary group $\mathrm{U}(n)$. In particular, the polynomial hull of $\mathrm{U}(n)$ is described. After introducing the open-cone condition and the closedcone condition, which bear some resemblance to the polydisc case, $\S 2$ contains the statement of all the results in this paper. Section 3 provides mainly the technical details for the proof of the results stated in $\S 2$. Finally, $\S 4$ contains some remarks that relate our work to that of Jimbo-Sakai [7] and that of Saerens [11], [12].

1. Definitions and certain properties of $\mathrm{U}(n)$. In this section, we are going to introduce several notations and definitions that we will 
use throughout the paper. Since we are mainly concerned with some analytic properties of $\mathrm{U}(n)$, we will ignore, for the most part, the algebraic structure of this compact Lie group.

Let $\mathrm{U}(n)$ be the unitary group in $C^{n^{2}}$, that is the set of all $n \times n$ unitary matrices. If we use the standard coordinates of $C^{n^{2}}$ and write $Z=\left(Z^{1}, \ldots, Z^{n}\right)^{t}$ with $Z^{i}=\left(Z_{1}^{i}, \ldots, Z_{n}^{i}\right)$, then

$$
\mathrm{U}(n)=\left\{Z \in C^{n^{2}}: \overline{Z^{t}} Z=Z \overline{Z^{t}}=I_{n}\right\} .
$$

The group $\mathrm{U}(n)$ is a real analytic manifold, and $\operatorname{dim}_{R} \mathrm{U}(n)=n^{2}$. If we define

$$
\begin{aligned}
& \phi_{j}^{i}(Z)=\operatorname{Re}\left\{\sum_{k=1}^{n} \overline{Z_{k}^{i}} Z_{k}^{j}-\delta_{j}^{i}\right\}, \quad 1 \leq i, j \leq n, \\
& \psi_{j}^{i}(Z)=\operatorname{Im}\left\{\sum_{k=1}^{n} \overline{Z_{k}^{i}} Z_{k}^{j}\right\}, \quad 1 \leq i, j \leq n,
\end{aligned}
$$

then $\mathrm{U}(n)=\left\{Z \in C^{n^{2}}: \phi_{j}^{i}(Z)=\psi_{j}^{i}(Z)=0\right.$, for all $\left.i, j\right\}$. Note that automatically we have $\psi_{i}^{i}(Z)=0$ for all $1 \leq i \leq n$. Under the identification of an $n^{2}$-vector $\xi=\left(\xi_{1}^{1}, \ldots, \xi_{n}^{1}, \ldots, \xi_{1}^{n}, \ldots, \xi_{n}^{n}\right)$ with an $(n \times n)$-matrix $\xi$ with row-vectors $\xi^{i}=\left(\xi_{1}^{i}, \ldots, \xi_{n}^{i}\right)$, the real tangent space of $\mathrm{U}(n)$ at a point $P$ is given by

$$
T_{P}(\mathrm{U}(n))=\left\{\xi \in C^{n^{2}}: \xi+P \overline{\xi t} P=0\right\} .
$$

This can be seen as follows. First, by the definition of real tangent space,

$$
\begin{aligned}
& T_{I_{n}}(\mathrm{U}(n))=\left\{\xi \in C^{n^{2}}:\left.\operatorname{Re}\left(\sum_{1 \leq 4 s, t \leq n} \xi_{t}^{s} \frac{\partial \phi_{j}^{i}}{\partial Z_{t}^{s}}\right)\right|_{Z=I_{n}}\right. \\
&=\left.\operatorname{Re}\left(\sum_{1 \leq s, t \leq n} \xi_{t}^{s} \frac{\partial \psi_{j}^{i}}{\partial Z_{t}^{s}}\right)\right|_{Z-I_{n}}=0, \\
&\quad \text { for all } 1 \leq i, j \leq n\} \\
&=\left\{\xi \in C^{n^{2}}: \xi+\overline{\xi^{t}}=0\right\} .
\end{aligned}
$$

If $P \in \mathrm{U}(n)$, so that $P: C^{n^{2}} \rightarrow C^{n^{2}}$ defined by $P(z)=P \cdot Z$ with the canonical correspondence between $C^{n^{2}}$ and $\operatorname{GL}(n, C)$ given above, 
then the mapping $P$ is nonsingular and linear. It carries $\mathrm{U}(n)$ to itself. The differential $d P: C^{n^{2}} \rightarrow C^{n^{2}}$ is just $P$, and $P I_{n}=P$. Thus

$$
T_{P}(\mathrm{U}(n))=d P\left(T_{I_{n}}(\mathrm{U}(n))\right)=\left\{\chi \in C^{n^{2}}: \chi=P \xi \text { with } \xi+\overline{\xi^{t}}=0\right\} .
$$

Then, as $\xi+\overline{\xi^{t}}=0$,

$$
0=\xi+\overline{\xi^{t}}=P^{-1} \chi+\overline{\left(P^{-1} \chi\right)^{t}}=P^{-1}\left(\chi+P \overline{\chi^{t}} P\right) .
$$

Our conclusion follows. (In particular, the tangent space of $\mathrm{U}(n)$ at the identity matrix may be identified with the set of all $n \times n$ skewhermitian matrices. That is to say, the Lie algebra of $\mathrm{U}(n)$ is the algebra of all skew-symmetric matrices.) Therefore $\mathrm{U}(n)$ is a totally real submanifold in $C^{n^{2}}$ with maximum dimension. The total reality of $\mathrm{U}(n)$ also follows from the observation that $\mathrm{U}(n)$ is the fixed submanifold of the antiholomorphic linear mapping $T: \operatorname{GL}(n, C) \rightarrow$ $\mathrm{GL}(n, C)$ given by $T(A)=\overline{\left(A^{-1}\right)^{t}}$.

Let $D$ be a bounded domain in $C^{n}$. We denote by $A^{k}(D)$ the algebra of all functions that are analytic on $D$ and have $k$ th $(0 \leq$ $k \leq \infty)$ continuous partial derivatives on the closure of $D$. The algebra $A^{\omega}$ consists of all analytic functions on $D$ that extend to be analytic on a neighborhood of $\bar{D}$. For the algebra $A^{0}$ (functions that are analytic on $D$ and continuous on $\bar{D})$, we write simply $A(D)$.

Given a bounded domain $D$ with smooth boundary $b D$, a compact subset $\Sigma$ is called a peak set for $A^{k}(D)$ if there is a function $f$ in $A^{k}(D)$ such that $\left.f\right|_{\Sigma}=1$ and $|f(z)|<1$ for all $z \in \bar{D} \backslash \Sigma$. A compact set $\Sigma \subset b D$ is called an interpolation set for $A^{k}(D)$ if for any given function $f \in C^{k}(\Sigma)$, there is an $F \in A^{k}(D)$ that coincides with $f$ on $\Sigma$. If $F$ can be chosen in such a way that $|F(w)|<\max _{z \in \Sigma}|F(z)|$ for all $w$ in $\bar{D} \backslash \Sigma$, then $\Sigma$ is called a peak-interpolation set for $A^{k}(D)$. We call $\Sigma$ a local peak set (local interpolation set, local peak-interpolation set) if for every point $p$ in $\Sigma$, the corresponding assumptions hold for $\Sigma \cap \bar{U}$ with some neighborhood $U$ of $p$.

Recall that for a compact subset $K$ in $C^{n}$, the polynomial hull of $K$ is defined to be

$$
\begin{aligned}
\widehat{K}=\left\{z \in C^{n},|p(z)| \leq\right. & \operatorname{Max}_{w \in K}|p(w)| \\
& \text { for all holomorphic polynomials } \left.p \text { in } C^{n}\right\} .
\end{aligned}
$$

In the following lemma, we use the notation $A \leq 0$ to mean that the square matrix $A$ has only nonpositive eigenvalues. 
LEMMA 1.1. The polynomial hull of the unitary group $\mathrm{U}(n)$ in $C^{n^{2}}$ is

$$
\widehat{\mathrm{U}(n)}=\left\{Z \in C^{n^{2}}: \overline{Z^{t}} Z-I_{n} \leq 0\right\} \text {. }
$$

Proof. Let $\Lambda=\left\{Z \in C^{n^{2}}, \overline{Z^{t}} Z-I_{n} \leq 0\right\}$. First we know that the torus $\Theta$ consisting of all matrices of the form $\operatorname{Diag}\left(e^{i \theta_{1}}, \ldots, e^{i \theta_{n}}\right)$ is contained in $\mathrm{U}(n)$. The polynomial hull of $\Theta$ is given by

$$
\widehat{\Theta}=\left\{\operatorname{Diag}\left(\lambda_{1}, \ldots, \lambda_{n}\right):\left|\lambda_{i}\right| \leq 1 \text { for } i=1, \ldots, n\right\} .
$$

On the other hand, every $n \times n$ matrix can be written as $U D V$ with $U, V$ two unitary matrices and $D$ a diagonal matrix, a direct consequence of Schur's lemma about diagonalizing square matrices (cf. [1, p. 195]). The set $\widehat{\mathrm{U}(n)}$ is invariant under the action of $\mathrm{U}(n)$. That is, if $P \in \widehat{\mathrm{U}(n)}$, then $U P V \in \widehat{\mathrm{U}(n)}$. Therefore $\Lambda \subseteq \widehat{\mathrm{U}(n)}$. Conversely, if $P \notin \Lambda$, then there is an eigenvalue of $P$, say $\lambda_{1}$, with modulus strictly greater than 1 . By Schur's lemma, there exists a unitary matrix $U$ such that $U^{-1} P U$ is an upper-triangle matrix with diagonal elements the eigenvalues of $P$. Assume that $\lambda_{1}$ appears in the first position of the diagonal and let $U^{-1}=\left(v_{j}^{i}\right)$. Then the polynomial

$$
p(Z)=\sum_{1 \leq s, t \leq n} u_{s}^{1} z_{t}^{s} v_{1}^{t}
$$

enjoys the following properties:

(1) $|p(P)|=\left|\lambda_{1}\right| \geq 1$,

(2) $\operatorname{Max}_{Z \in \mathrm{U}(n)}|p(Z)|=\operatorname{Max}_{Z \in \mathrm{U}(n)}\left|z_{1}^{1}\right| \leq 1$.

This completes the proof.

From the above proof, we can obtain a stronger conclusion that the linear convex hull of $\mathrm{U}(n)$ coincides with its polynomial hull, and therefore that $\widehat{\mathrm{U}(n)}$ is convex. Also, from the above lemma, $\widehat{\mathrm{U}(n)}$ is closed under matrix multiplication and has $\mathrm{U}(n)$ as its Shilov boundary. Since a Hermitian matrix is positive definite (i.e., all eigenvalues are positive) if and only if the determinant of all its principal minors are positive, $\widehat{\mathrm{U}(n)}$ is the semianalytic set defined by

$$
\begin{array}{r}
\widehat{\mathrm{U}(n)}=\left\{Z \in C^{n^{2}}: \operatorname{Det}\left[\delta_{j}^{i}-\sum_{k=1}^{n} \overline{z_{k}^{i}} z_{k}^{j}\right]_{1 \leq i, j \leq l}>0\right. \\
\text { for all } l=1, \ldots, n\} .
\end{array}
$$


2. Statement of results. In this section we will state several theorems; we leave the proofs to the next section.

Let $\mathscr{S}$ be the set of all skew-Hermitian matrices $A$ and define

$$
\mathscr{P}_{+}=\{A \in \mathscr{S}: i A>0\}, \quad \mathscr{P}_{-}=\{A \in \mathscr{S}: i A<0\} .
$$

A $C^{1}$ submanifold $\Sigma$ in $\mathrm{U}(n)$ is said to satisfy the open cone (resp. closed cone) condition if the following holds for every point $p \in \Sigma$

$$
T_{p}(\Sigma) p^{-1} \subset \mathscr{S} \backslash \overline{\mathscr{P}_{+} \cup \mathscr{P}_{-}}
$$

(resp. if $T_{p}(\Sigma) p^{-1} \subseteq \mathscr{S} \backslash\left(\mathscr{P}_{+} \cup \mathscr{P}_{-}\right)$).

Here we are using the identification of an $n^{2}$-vector $\xi$ with an $n \times n$ matrix as we mentioned at the beginning of $\S 1$. For a $k$-dimensional $C^{1}$ submanifold $\Sigma$ in $C^{n^{2}}$, the real tangent space at the point $p$ is defined by

$$
\begin{aligned}
T_{p}(\Sigma) & =\left\{J(\Phi) p \cdot V: V \in R^{k}\right\} \\
& =\left\{\left(\left.\sum_{l=1}^{k} \frac{\partial \phi_{t}^{s}}{\partial x_{l}}\right|_{p} v^{l}\right)_{1 \leq s, t \leq n}: V=\left(v^{1}, \ldots, v^{k}\right) \in R^{k}\right\},
\end{aligned}
$$

where $\Sigma$ is parameterized near $p$ by the $C^{1}$ mapping

$$
\boldsymbol{\Phi}=\left(\phi_{t}^{s}\right)_{1 \leq s, t \leq n}: R^{k} \rightarrow C^{n^{2}}
$$

and $J(\Phi)_{p}$ is the $n^{2} \times k$ Jacobian of $\Phi$ at $p$. Therefore our open cone (or closed cone) condition simply means that for all $V=\left(v^{1}, \ldots, v^{k}\right)$ in $R^{k}$, the matrix

$$
i\left(\left.\sum_{l=1}^{k}\left(\sum_{r=1}^{n} \frac{\partial \phi_{r}^{s}}{\partial x_{l}} \overline{\phi_{r}^{t}}\right)\right|_{p} v^{l}\right)_{1 \leq s, t \leq n}
$$

is neither positive semi-definite nor negative semi-definite (or neither positive definite nor negative definite).

Our first theorem is a simple analogue of [2, Theorem IV.1] and follows from the semianalyticity of $\widehat{\mathrm{U}(n)}$.

THEOREM 2.1. A real-analytic closed submanifold $\Sigma$ in $\mathrm{U}(n)$ is an interpolation set for $A^{\omega}(\widehat{\mathrm{U}(n)})$ if and only if there exists a complex submanifold $\mathscr{M}$ in a neighborhood of $\widehat{\mathrm{U}(n)}$ such that

$$
\Sigma=\mathrm{U}(n) \cap \mathscr{M}=\widehat{\mathrm{U}(n)} \cap \mathscr{M} .
$$

There is a geometric description of the real-analytic submanifolds that are interpolation sets for $A^{\omega}(\widehat{\mathrm{U}(n)})$ : 
THEOREM 2.2. Let $\Sigma$ be a real analytic closed submanifold of $\mathrm{U}(n)$. If $\Sigma$ satisfies the open cone condition at each of its points, then $\Sigma$ is an interpolation set for $A^{\omega}(\widehat{\mathrm{U}(n)})$. Conversely, if $\sigma$ is an interpolation set or $A^{\omega}(\widehat{\mathrm{U}(n)})$, then $\Sigma$ necessarily satisfies the closed cone condition at each point.

For the $C^{0}$-interpolation problem, we can get a stronger result as follows

THEOREM 2.3. Let $\Sigma$ be a closed $C^{2}$ submanifold in $\mathrm{U}(n)$. If $\Sigma$ satisfies the open cone condition, then $\Sigma$ is a peak-interpolation set for $A(\widehat{\mathrm{U}(n)})$. Conversely, $\Sigma$ must satisfy the closed cone condition if it is $a$ peak-interpolation for $A(\widehat{\mathrm{U}(n)})$.

In the case of $C^{\infty}$, we have obtained only a partial result as follows. For the corresponding results in the polydisc case, see [8].

THEOREM 2.4. Suppose that $\Sigma$ is a closed $C^{\infty}$ submanifold of $\mathrm{U}(n)$. If $\Sigma$ satisfies the open cone condition at each of its points, then $\Sigma$ is $a$ local peak set and also an interpolation set for $A^{\infty}(\widehat{\mathrm{U}(n)})$. Conversely, if $\Sigma$ is a peak set for $A^{k}(\widehat{\mathrm{U}(n)})$ with $k \geq 1$, then there exists an $\left(n^{2}-1\right)$-dimensional $C^{k}$ submanifold $\mathscr{M}$ in some neighborhood of $\widehat{\mathrm{U}(n)}$ such that $\Sigma \subset \mathscr{M} \cap \mathrm{U}(n)$ and $\mathscr{M} \cap \mathrm{U}(n)$ satisfies the closed cone condition at each of its points.

We end this section by giving some examples.

EXAMPLE 2.5. Consider the real analytic curve $\Gamma$ in $\mathrm{U}(n)$ defined by

$$
\Gamma(t)=\left(\begin{array}{cc}
\varepsilon e^{(n+i) t} & \sqrt{1-\varepsilon^{2} e^{2 \eta t}} \\
-\sqrt{1-\varepsilon^{2} e^{2 \eta t}} & \varepsilon e^{(\eta-i) t}
\end{array}\right)
$$

where $\sqrt{2} / 2<\varepsilon<1$ and $1+\eta^{2}-3 \varepsilon^{2}+2 \varepsilon^{4}<0$. Then we have

$$
i \Gamma^{\prime}(0) \overline{\Gamma(0)^{t}}=\left(\begin{array}{cc}
-\varepsilon^{2} & \frac{\varepsilon-\varepsilon^{3}-i \varepsilon \eta}{\sqrt{1-\varepsilon^{2}}} \\
\frac{\varepsilon-\varepsilon^{3}+i \varepsilon \eta}{\sqrt{1-\varepsilon^{2}}} & \varepsilon^{2}
\end{array}\right) .
$$

Hence the curve is a peak-interpolation set for $A(\widehat{\mathrm{U}(n)})$ for $t$ near 0 , since at $t=0$ we have that

$$
\operatorname{Det}\left(i \Gamma^{\prime}(0) \Gamma(\overline{0})^{t}\right)=\frac{\varepsilon^{2}}{1-\varepsilon^{2}}\left(1+\eta^{2}-3 \varepsilon^{2}+2 \varepsilon^{4}\right)<0
$$


EXAMPLE 2.6. Let $\mathrm{SU}(n)$ be the special unitary group in $\mathrm{U}(n)$. Then $\mathrm{SU}(n)$ is a peak-interpolation set for $A^{\omega}(\widehat{\mathrm{U}(n)})$. This can be seen either from Theorem 2.1, since

$$
\begin{aligned}
\mathrm{SU}(n) & =\mathrm{SU}(n) \cap\left\{Z \in C^{n^{2}}: \operatorname{det}(Z)=1\right\} \\
& =\widehat{\mathrm{U}(n)} \cap\left\{Z \in C^{n^{2}}: \operatorname{det}(Z)=1\right\}
\end{aligned}
$$

and $\left\{Z \in C^{n^{2}}: \operatorname{det}(Z)=1\right\}$ is a complex submanifold in $C^{n^{2}}$. Alternatively, the result follows from Theorem 2.2 , since for any $\xi \in$ $T_{P}(\mathrm{SU}(n))$, we have that

$$
\operatorname{Tr}\{i \xi P\}=0
$$

and this implies that $\xi$ can be neither positive definite nor negative definite. For any fixed real number $\theta$, we define a submanifold

$$
\mathrm{U}_{\theta}(n)=\left\{Z \in U(n): \operatorname{det}(Z)=e^{i \theta}\right\} .
$$

Then $\mathrm{U}_{\theta}$ is a peak-interpolation set for $A^{\omega}(\widehat{\mathrm{U}(n)})$ and for $A(\widehat{\mathrm{U}(n)})$, since we can show that this submanifold satisfies the open cone condition. Similar to the second proof, one can show that the special orthogonal group

$$
\mathrm{SO}(n)=\left\{Z \in R^{n^{2}}: Z^{t} Z=I_{n}\right\}
$$

is also a peak-interpolation set for $A^{\omega}(\widehat{\mathrm{U}(n)})$ and for $A(\widehat{\mathrm{U}(n)})$.

3. The proof of the theorems. In this section, we will give a complete proof of the theorems stated in the last section. The ideas in these proofs are originally from Nagel and Rudin [9], Burns and Stout [2], and Saerens [11]. Two preliminary results from matrix analysis will be used to deal with $\mathrm{U}(n)$.

LEMMA 3.1. If $A=\left(a_{j}^{i}\right)$ is an $n \times n$ positive definite matrix and $B=\left(b_{j}^{i}\right)$ is an $n \times n$ negative definite matrix, then we have following inequality:

$$
\sum_{i, j=1}^{n} a_{j}^{i} b_{j}^{i}<0 .
$$

Proof. Recall that the Schur product of two $n \times n$ square matrices $A=\left(a_{j}^{i}\right)$ and $B=\left(b_{j}^{i}\right)$ is the $n \times n$ matrix $C=\left(c_{j}^{i}\right)$ defined by $c_{j}^{k}=a_{j}^{i} b_{j}^{i}$. It is well known that the Schur product of two positive definite matrices is still positive definite (cf. [1, p. 94]). Therefore 
the matrix $C=\left(a_{j}^{i} b_{j}^{i}\right)$ is negative definite. Taking $X=(1, \ldots, 1)$, $X C \overline{X^{t}}<0$ gives our first lemma.

LEMMA 3.2. If an $n \times n$ matrix $A=\left(a_{j}^{i}\right)$ is positive definite, then the $n^{2} \times n^{2}$ matrix $B=\left(a_{j}^{i} I_{n}\right)$ is also positive definite.

Proof. Let $X=\left(X_{1}, \ldots, X_{n}\right)$ be any vector in $C^{n^{2}}$ with $X_{i}=$ $\left(X_{i}^{1}, \ldots, X_{i}^{n}\right)$ in $C^{n}$, and let $B_{j}^{i}=\left(a_{j}^{i} I_{n}\right)_{n \times n}$. Then $B=\left(B_{j}^{i}\right)$. Now

$$
\begin{aligned}
X B \overline{X^{t}} & =\sum_{i, j=1}^{n} X_{i} B_{j}^{i} \overline{X_{j}^{t}}=\sum_{i, j=1}^{n} \sum_{k, l=1}^{n} a_{j}^{i} X_{i}^{k} \overline{X_{j}^{l}} \\
& =\sum_{k, l=1}^{n}\left(\sum_{i, j=1}^{n} a_{j}^{i} X_{i}^{k} \overline{X_{j}^{l}}\right) .
\end{aligned}
$$

Since $A>0$, for any fixed $k, l$, every term inside the parentheses is strictly positive. Therefore $B$ is strictly positive definite.

The proof of Theorem 2.1 follows, mutatis mutandis, from the algebraic proof of Theorem IV.1 of [2], since we know that $\widehat{U(n)}$ is a semi-analytic set and that the polynomial hull of $\mathrm{U}(n), \widehat{\mathrm{U}(n)}$ has a Stein neighborhood system.

The proof of Theorem 2.2 consists of two parts. First we prove the theorem for 1-dimensional submanifolds, i.e., for curves, as follows.

Proposition 3.3. Let $\Gamma$ be a simple closed real analytic curve in $\mathrm{U}(n)$, say $\Gamma=\Gamma(R)$ with $\Gamma(t)$ real analytic, regular and injective on $[-1,1)$, and periodic with period 2. If for every $t \in R$, we have that

$$
\Gamma^{\prime}(t) \overline{\Gamma(t)^{t}} \subset \mathscr{S} \backslash \overline{\mathscr{P}_{+} \cup \mathscr{P}_{-}},
$$

then $\Gamma$ is an interpolation submanifold of $A^{\omega} \widehat{\mathrm{U}(n)}$. Conversely, if $\Gamma$ is a closed interpolation submanifold of $A^{\omega} \widehat{\mathrm{U}(n)}$, then for all $t \in R$

$$
\Gamma^{\prime}(t) \overline{\Gamma(t)^{t}} \subset \mathscr{S} \backslash \mathscr{P}_{+} \cup \mathscr{P}_{-} .
$$

Proof. Let $I=[-1,1]$. Then $\Gamma(1)=\Gamma(-1)$ and $\Gamma=\Gamma(I)$. We can assume, without loss generality, that $\Gamma(0)=I_{n}$. Since $\Gamma$ is real analytic in a neighborhood of the interval $I$ and is periodic, we can extend $\Gamma$ to be holomorphic in a neighborhood $R \times[-\eta, \eta] \subset C^{1}$ of $R$ for some suitable $\eta>0$. We denote this extension again by $\Gamma$. 
We have, by the Taylor expansion of $\Gamma(t+i s)$ about a point $t \in R$, that for small $s$

$$
\Gamma(t+i s)=\Gamma(t)+i s \Gamma^{\prime}(t)-\frac{s^{2}}{2} \Gamma^{\prime \prime}(t)+o\left(s^{2}\right) .
$$

As $\Gamma(R) \subset \mathrm{U}(n)$, we have

$$
\Gamma(t) \overline{\Gamma(t)^{t}}=I_{n}
$$

for all $t \in I$. Therefore, by differentiating the above equality with respect to $t$, we obtain

$$
\begin{gathered}
\Gamma^{\prime}(t) \overline{\Gamma(t)^{t}}+\Gamma(t) \overline{\Gamma^{\prime}(t)^{t}}=0, \\
\Gamma^{\prime \prime}(t) \overline{\Gamma(t)^{t}}+2 \Gamma^{\prime}(t) \overline{\Gamma^{\prime}(t)^{t}}+\Gamma(t) \overline{\Gamma^{\prime \prime}(t)^{t}}=0 .
\end{gathered}
$$

According to these equalities, we get

$$
\begin{aligned}
I_{n}- & \Gamma(t+i s) \overline{\Gamma(t+i s)^{t}} \\
= & i s\left(\Gamma(t) \overline{\Gamma^{\prime}(t)^{t}}-\Gamma^{\prime}(t) \overline{\Gamma(t)^{t}}\right) \\
& +\frac{s^{2}}{2}\left(\Gamma^{\prime \prime}(t) \overline{\Gamma(t)^{t}}+\Gamma(t) \overline{\Gamma^{\prime \prime}(t)^{t}}-2 \Gamma^{\prime}(t) \overline{\Gamma^{\prime}(t)^{t}}\right)+o\left(s^{2}\right) \\
= & 2 i s \Gamma(t) \overline{\Gamma^{\prime}(t)^{t}}-2 s^{2} \Gamma^{\prime}(t) \overline{\Gamma^{\prime}(t)^{t}}+o\left(s^{2}\right) .
\end{aligned}
$$

If $\Gamma^{\prime}(t) \overline{\Gamma(t)^{t}} \notin \overline{\mathscr{P}_{+} \cup \mathscr{P}_{-}}$, then the matrix $\Gamma^{\prime}(t) \overline{\Gamma(t)^{t}}$ has two eigenvalues with opposite signs. Therefore, there exists a constant $\sigma>0$ such that for every $t \in R$, the matrix $I_{n}-\Gamma(t+i s) \overline{\Gamma(t+i s)^{t}}$ also has two eigenvalues with opposite signs for $t \in R$ and $|s|<\sigma$, i.e., for these $t$ and $s$, the matrix $I_{n}-\Gamma(t+i s) \overline{\Gamma(t+i s)^{t}}$ cannot be positive semi-definite or negative semi-definite. Define $\widetilde{\Gamma}$, a complexification of $\Gamma$, by

$$
\widetilde{\Gamma}=\{\Gamma(z): z=t+i s \text { with } t \in R \text { and }|s|<\sigma\} .
$$

Note that $\widetilde{\Gamma}$ is a submanifold of a neighborhood of $\mathrm{U}(n)$, provided that $\sigma$ is small enough. The above observation implies that $\widetilde{\Gamma}$ meets $\widehat{\mathrm{U}(n)}$ only along $\mathrm{U}(n)$, i.e.,

$$
\widetilde{\Gamma} \cap \widehat{\mathrm{U}(n)}=\widetilde{\Gamma} \cap \mathrm{U}(n)=\Gamma \text {. }
$$

Therefore our conclusion that $\Gamma$ is an interpolation set for $A^{\omega}(\widehat{\mathrm{U}(n)})$ follows from Theorem 2.1.

Conversely, suppose $\Gamma^{\prime}\left(t_{0}\right) \overline{\Gamma\left(t_{0}\right)^{t}} \in \mathscr{P}_{+} \cup \mathscr{P}_{-}$for some $T_{0} \in R$. We assume that $t_{0}=0$ and $\Gamma\left(t_{0}\right)=I_{n}$. Then there exists a small neighborhood $J=[-\eta, \eta]$ of 0 so that for all $t \in J$, the matrix 
$\Gamma^{\prime}(t) \overline{\Gamma(t)^{t}}$ is either positive definite or negative definite, say the former. Now the positive definiteness of this matrix implies that we can find a positive constant $\sigma$ such that the following two conditions hold for $D=\{z=t+i s: t \in J$ and $|s|<\sigma\}$ :

1. The matrix $\Gamma^{\prime}(z)$ is nonsingular for $z \in D$.

2. Then the open set $D$ is mapped by $\Gamma$ to $C^{n^{2}}$ as follows:

$$
D_{-}=\{z=t+i s, t \in J \text { and }-\sigma<s \leq 0\} \rightarrow C^{n^{2}} \backslash(\widehat{\mathrm{U}(n)} \backslash \mathrm{U}(n)),
$$

and

$$
D_{+}=\{z=t+i s, t \in J \text { and } 0 \leq s<\sigma\} \rightarrow \widehat{\mathrm{U}(n)},
$$

or the other way around, $D_{-}$is mapped to $\widehat{\mathrm{U}(n)}$ and $D_{+}$to $C^{n^{2}} \backslash(\widehat{\mathrm{U}(n)} \backslash \mathrm{U}(n))$. We assume the former one.

Therefore the embedded complex curve $\Gamma\left(D_{-}\right)$meets $\widehat{\mathrm{U}(n)}$ only along the curve $\Gamma$, while the embedded complex curve $\Gamma\left(D_{+}\right)$is contained entirely in $\widehat{\mathrm{U}(n)}$ and meets $\mathrm{U}(n)$ only along $\Gamma$. The variety $\widetilde{\Gamma}$ is a one-dimensional complex manifold that is biholomorphically equivalent to an annulus in the complex plane, provided $\sigma>0$ is small enough. There is a function $\phi$ defined and holomorphic on a neighborhood of $\Gamma$ in $\widetilde{\Gamma}$ that takes $\Gamma$ bianalytically onto the unit circle in $C$. We can suppose that $\phi \circ \Gamma$ takes $D_{+}$into the unit disc in $C$ and takes $D_{-}$into the complement of the unit disc. If $z_{0} \in C$ lies in the unit disc but has modulus very near one, then the function $f$ defined on $\Gamma$ by

$$
f(w)=\frac{1}{\phi(w)-z_{0}}
$$

is in $C^{\omega}(\Gamma)$, but does not extend analytically to $\Gamma\left(D_{+}\right)$. This gives the proof of the second part of the proposition.

The above proposition leads to the proof of Theorem 2.2, by reducing the dimension of $\Sigma$ to 1 as follows:

Suppose that $\Sigma$ is an interpolation set for $A^{\omega}(\widehat{\mathrm{U}(n)})$, i.e.,

$$
\left.A^{\omega}(\widehat{\mathrm{U}(n)})\right|_{\Sigma}=C^{\omega}(\Sigma) \text {, }
$$

but there is a point $P$ in $\Sigma$ such that $T_{P}(\Sigma) \overline{P^{t}}$ is not contained in $\mathscr{S} \backslash\left(\mathscr{P}_{+} \cup \mathscr{P}_{-}\right)$. Then we can find an $n \times n$ matrix $v_{P}$ in $T_{P}(\Sigma)$, so that $v_{P} \overline{P^{t}} \in \mathscr{P}_{+} \cup \mathscr{P}_{-}$, say it is positive definite. There exists a simple closed analytic curve $\Gamma$ in $\Sigma$ in a neighborhood of $P$ such that $\Gamma(1)=P$ and $\Gamma^{\prime}(t)=v_{P}$. Here we identify the curve $\Gamma$ with 
the image of real analytic map $\Gamma$ from the unit circle $S^{1}$ in $C$. The mapping $\Gamma$ can be extended to a holomorphic function in a tubular neighborhood $D$ of $S^{1}$. The positiveness of the matrix $v_{P} \overline{P^{t}}$ implies that there exists a neighborhood $B$ of $(1,0)$ so that one of the sets $D_{-}=\{|z|<1\} \cap D \cap B$ and $D_{+}=\{|z|>1\} \cap D \cap B$ is mapped into $\widehat{\mathrm{U}(n)}$ and the closure of the image meets $\mathrm{U}(n)$ only along $\Gamma$. Thus, by the method we used in the proof of the second part of the above proposition, a real analytic function $f$ on $\Gamma$ can be obtained that cannot be extended to a neighborhood of $\Gamma$ inside $\widehat{U(n)}$. Since $\Sigma$ is a simple closed real analytic curve, $C^{\omega}(\Gamma)=\left.C^{\omega}(\Sigma)\right|_{\Gamma}$, which leads to the contradiction that $C^{\omega}(\Sigma) \neq\left. A^{\omega}(\widehat{\mathrm{U}(n)})\right|_{\Sigma}$. This contradiction yields the necessary condition in Theorem 2.2.

To prove the sufficiency, we only need to construct a complexification $\widetilde{\Sigma}$ of $\Sigma$ that intersects $\mathrm{U}(n)$ in $\Sigma$. Suppose $\operatorname{dim}_{R} \Sigma=m$. Then there exists a bianalytic map $\Phi$ from an open set $\Omega$ in $R^{m}$ to a neighborhood $B$ of the point $P$ such that $\Sigma \cap B$ is the image of $\Phi$. The open cone condition of $\Sigma$ means that the matrix

$$
i\left(\sum_{l=1}^{m} \sum_{r=1}^{n} \frac{\partial \Phi_{r}^{s}}{\partial x_{l}} \overline{\Phi_{r}^{t}} v^{l}\right)_{1 \leq s, t \leq n}
$$

is neither positive definite nor negative definite for all points in $\Omega$ and for all $V=\left(v^{1}, \ldots, v^{n}\right)$. Since $\Phi$ is real analytic on $\Omega$, we can choose a thin neighborhood $\widetilde{\Omega}$ of $\Omega$ in $C^{m}$ and extend $\Phi$ to an analytic mapping, again denoted by $\Phi$, from $\widetilde{\Omega}$ to a complex submanifold $\widetilde{\Sigma}$ in $C^{n^{2}}$. For each fixed $X_{0}+i Y_{0}$ in $\widetilde{\Omega}$, consider the curve

$$
\Gamma(t)=\Phi\left(X_{0}+i t Y_{0}\right), \quad \text { for }|t|<1 .
$$

(We can assume that $\widetilde{\Omega}$ is symmetric about $R^{m}$.) For all $t$ in the unit interval, we are in the same situation as we had in the proof of the first part in Proposition 3.3. Hence by choosing small $\widetilde{\Omega}$, we can obtain a complex submanifold $\widetilde{\Sigma}$ contained in $\widetilde{\Omega}$ that meets $\widehat{\mathrm{U}(n)}$ only along the $\Sigma$. This completes our proof of Theorem 2.2

In order to prove Theorem 2.3, first we make the following convention:

Given two vectors in $C^{n^{2}}=R^{2 n^{2}}$. We regard them as two $n \times n$ matrices $U=\left(u_{s t}^{1}+i u_{s t}^{2}\right)$ and $V=\left(v_{s t}^{1}+i v_{s t}^{2}\right)$ and define the usual Euclidean inner product between these two vectors (matrices) as

$$
U \cdot V=\sum_{s, t=1}^{n}\left(u_{s t}^{1} v_{s t}^{1}+u_{s t}^{2} v_{s t}^{2}\right)
$$


This is the real part of the standard Hermitian inner product in $C^{n^{2}}$; it is the standard real inner product on $C^{n^{2}}=R^{2 n^{2}}$.

Recall that a $C^{1}$ submanifold $\mathscr{M}$ in $C^{n}$ is called totally real at a point $P$ in $\mathscr{M}$ if there is no complex subspace contained in the real tangent space of $\mathscr{M}$ at $P$, except the origin. Harvey and Wells [3] proved that if $M$ is a $C^{1}$ totally real submanifold of a complex manifold $X$, then there exist a neighborhood $\mathscr{N}$ of $M$ and a nonnegative function $\phi \in C^{2}(\mathscr{N})$ such that

1. $M=\{z \in \mathscr{N}: \phi(z)=0\}=\{z \in X: \operatorname{grad}(\phi(z))=0\}$,

2. $\phi(z)$ is strictly plurisubharmonic on $\mathscr{N}$.

The idea for proving Theorem 2.3 is, at every point $P$ in $\Sigma$, to embed $\Sigma$ locally into the boundary of a strictly pseudoconvex domain $\mathscr{D}$ in $C^{n^{2}}$ such that $\Sigma$ is complex tangential in the boundary $b \mathscr{D}$. This can be done first by requiring $\mathscr{D}$ to be strictly pseudoconvex near the point $P$. Then use a method from Stout [13] and Saerens [11] to get a globally strictly pseudoconvex domain $\mathscr{D}$ so that $\Sigma$ is also complex tangential near $P$. Thus $\Sigma$ is a local peak set and a local interpolation set for $A(\widehat{\mathrm{U}(n)})$, following results from Henkin-Tumanov [5]. Moreover, by using partition of unity exactly as done in Hakim-Sibony [4], $\Sigma$ is an interpolation set for $A(\widehat{\mathrm{U}(n)})$. Finally the property of global peak-interpolation for $\Sigma$ is obtained from Varopoulos' Theorem in [14]. We give the proof of the first part and leave the proof of the second part to the references [11] and [13]. Without loss of generality, we can assume that $P=I_{n}$.

Proposition 3.4. Let $\Sigma$ be a $C^{2}$ submanifold of $\mathrm{U}(n)$ that contains $I_{n}$. If $\Sigma$ satisfies the open cone condition at $I_{n}$, then there exists a $C^{2}$ function $\rho: C^{n^{2}} \rightarrow R$ such that for the domain $\mathscr{D}$ defined by $\mathscr{D}=\left\{Z \in C^{n^{2}}: \rho(Z)<0\right\}$, there is an open subset $\mathscr{U}$ in $C^{n^{2}}$ such that

1. $d \rho(Z) \neq 0$ for $Z$ in $\mathscr{U}$,

2. $\mathrm{U}(n) \cap \mathscr{U} \subset b \mathscr{D}$ and $\mathscr{U} \cap(\widehat{\mathrm{U}(n)} \backslash \mathrm{U}(n)) \subset \mathscr{D}$,

3. $T_{P}(\Sigma) \subset T_{P}^{C}(b \mathscr{D})$ if $P \in \mathscr{U}$, and

4. $\rho$ is strictly plurisubharmonic in a neighborhood of $b \mathscr{D} \cap \mathscr{U}$.

Proof. Assume $\operatorname{dim}_{R} \Sigma=m$. Then $m \leq n^{2}-1$. We note that there exists a neighborhood $B$ of the point $I_{n}$ in $\mathrm{U}(n)$ and an $\left(n^{2}-1\right)$ dimensional $C^{2}$ submanifold $\widetilde{\Sigma}$ of $\mathrm{U}(n)$ so that $\widetilde{\Sigma} \cap B$ satisfies the open cone condition and contains $\Sigma \cap B$. This can be done, using the equivalent definition of cone conditions stated in Remark 4.1, by the 
same argument given in Saerens [11]. (See the proof of Lemma IV.2 in [11].)

So starting from now, we can assume that the manifold $\Sigma$ is of top dimension, i.e., of dimension $n^{2}-1$. We need to show that if

$$
T_{P}(\Sigma) \overline{P^{t}} \subset \mathscr{S} \backslash\left(\overline{\mathscr{P}_{+} \cap \mathscr{P}_{-}}\right)
$$

for $P \in \Sigma$ near the identity, then a normal vector $e(P) \in\left(T_{P}(\Sigma) \overline{P^{t}}\right)^{\perp}$ can be chosen such that $e(P) \in \mathscr{P}_{+} \cup \mathscr{P}_{-}$. For this, it is enough to assume that $P=I_{n}$ and that $\Sigma$ is given by $\Phi(Z)=0$ for $Z \in \mathrm{U}(n)$ near $I_{n}$ and $\Phi$ a $C^{2}$ real-valued function with $d \Phi \neq 0$ on $\Sigma$.

Let $B_{\Phi}$ be the skew-hermitian matrix defined by

$$
B_{\Phi}=\left(B_{i j}\right)=\left(\left(\frac{\partial \Phi}{\partial Z_{j}^{i}}-\frac{\partial \Phi}{\partial \overline{Z_{i}^{j}}}\right)\left(I_{n}\right)\right) \text {. }
$$

Then there exists a unitary matrix $U$ so that

$$
U B_{\Phi} \overline{U^{t}}=i \operatorname{diag}\left(\lambda_{1}, \ldots, \lambda_{n}\right), \quad \lambda_{i} \in R .
$$

Consider a linear change of coordinates $L: Z \rightarrow \bar{U} Z U^{t}$. Then under this mapping, $\mathrm{U}(n) \rightarrow \mathrm{U}(n), I_{n} \rightarrow I_{n}$, and for $Z \in L(\mathrm{U}(n))=\mathrm{U}(n)$ near the identity, $\Sigma$ is given by $\Psi(Z)=\Phi\left(U^{t} Z \bar{U}\right)=0$. Moreover,

$$
\begin{aligned}
& \frac{\partial \Psi}{\partial Z_{j}^{i}}=\sum_{k, l} U_{k}^{i} \frac{\partial \Phi}{\partial Z_{l}^{k}} \overline{U_{l}^{j}}, \\
& \frac{\partial \psi}{\partial \overline{Z_{i}^{j}}}=\sum_{k, l} \leq U_{k}^{j} \frac{\partial \Phi}{\partial \overline{Z_{l}^{k}}} U_{l}^{i}=\sum_{k, l} U_{k}^{i} \frac{\partial \Phi}{\partial \overline{Z_{k}^{l}}} \overline{U_{l}^{j}} .
\end{aligned}
$$

Thus

$$
B_{\Psi}=U B_{\Phi} \overline{U^{t}}=i \operatorname{diag}\left(\lambda_{1}, \ldots, \lambda_{n}\right) .
$$

On the other hand, by the definition of the real tangent space,

$$
T_{I_{n}}(\Sigma)=\left\{A=\left(A_{j}^{i}\right) \in \mathscr{S}: \operatorname{Re}\left\{\left.\sum_{i, j} A_{j}^{i} \frac{\partial \Psi}{\partial Z_{j}^{i}}\right|_{I_{n}}\right\}=0\right\} \text {. }
$$

If $A=-\overline{A^{t}}$, then

$$
\begin{aligned}
\operatorname{Re}\left\{\left.\sum_{i, j} A_{j}^{i} \frac{\partial \Psi}{\partial Z_{j}^{i}}\right|_{I_{n}}\right\} & =\frac{1}{2} \sum_{i, j} A_{j}^{i}\left(\left.\left(\frac{\partial \Psi}{\partial Z_{j}^{i}}-\frac{\partial \Psi}{\partial \overline{Z_{i}^{j}}}\right)\right|_{I_{n}}\right) \\
& =\frac{1}{2} \sum_{i, j} A_{j}^{i}\left(B_{\Psi}\right)_{j}^{i}=\frac{i}{2} \sum_{i} \lambda_{i} A_{i}^{i} .
\end{aligned}
$$


Therefore, the condition $T_{I_{n}}(\Sigma) \subset \mathscr{S} \backslash \overline{\mathscr{P}_{+} \cup \mathscr{P}_{-}}$implies that the real numbers $\lambda_{1}, \ldots, \lambda_{n}$ all have the same sign. (For if, say, $\lambda_{1}>0$ and $\lambda_{2}<0$, then the skew-hermitian matrix $A=i \operatorname{diag}\left(\lambda_{2},-\lambda_{1}, 0, \ldots, 0\right)$ would be in $\overline{\mathscr{P}}_{+} \cap T_{I_{n}}(\Sigma)$.) Thus the matrix $i B_{\Psi}$ is either positive definite or negative definite, and by our definition of normal vector, we can choose $e\left(I_{n}\right)=B_{\Psi}$, since for all vectors $A \in T_{I_{n}}(\Sigma)$,

$$
A \cdot e\left(I_{n}\right)=\operatorname{Re} \sum_{i, j} A_{j}^{i}\left(B_{\Psi}\right)_{j}^{i}=0 .
$$

For a general point $P=\left(P_{j}^{i}\right) \in \Sigma$ near $I_{n}$, we can choose

$$
e(P)=\left(\left(\frac{\partial \Phi}{\partial Z_{k}^{i}}-\frac{\partial \Phi}{\partial \overline{Z_{i}^{k}}}\right)(P) P_{k}^{j}\right),
$$

and the positive (or negative) definiteness of $e(P)$ follows from that of $e\left(I_{n}\right)$.

To construct our desired function $\rho$, we assume, without loss of generality, that $e(P) \in \mathscr{P}_{+}$; i.e., the matrix $i e(P)=\left(C_{l}^{k}(P)+i D_{l}^{k}(P)\right)$ is positive definite for $P$ near the identity.

By the theorem of Harvey and Wells, there exist a neighborhood $\mathscr{N}$ of $\Sigma$ and a non-negative strictly plurisubharmonic function $\phi(Z)$ in $\mathscr{N}$ such that

$$
\Sigma=\{Z \in \mathscr{N}: \phi(Z)=0\}=\{Z \in \mathcal{N}: \operatorname{grad} \phi(Z)=0\} .
$$

If $H(\phi)_{P}$ denotes the complex Hessian of $\phi(Z)$ at $P$, i.e.,

$$
H(\phi)_{P}=\left(\frac{\partial^{2} \phi}{\partial \zeta_{j} \partial \zeta_{k}}(P)\right)
$$

where $1 \leq j, k \leq n^{2}$ and $\zeta_{j}$ runs through the $n^{2}$ complex coordinates $Z_{j}^{i}$, then the $n^{2} \times n^{2}$ matrix $H(\phi)_{Z}$ is positive definite near $I_{n}$. Therefore there exists a large positive number $\alpha_{0}$ such that the $n^{2} \times n^{2}$ Hermitian matrix $B+\alpha_{0} H(\phi)_{I_{n}}$ is positive definite, where

$$
B=\left.\left(\frac{\partial\left(C_{t}^{s}(Z)+i D_{t}^{s}(Z)\right)}{\partial Z_{v}^{u}}\right)\right|_{Z=I_{n}}+\left.\left(\frac{\overline{\partial C_{t}^{s}(Z)+i D_{t}^{s}(Z)}}{\partial Z_{v}^{u}}\right)^{t}\right|_{Z=I_{n}}
$$

We define a real-valued $C^{2}$ function

$$
\rho_{1}(Z)=\sum_{s, t=1}^{n}\left(C_{t}^{s}(Z) \varphi_{t}^{s}(Z)+D_{t}^{s}(Z) \psi_{t}^{s}(Z)\right)
$$

where $\left\{\varphi_{t}^{S}(Z), \psi_{t}^{S}(Z)\right\}$ are the defining functions of $\mathrm{U}(n)$ defined 
in $\S 1$, and choose an arbitrary $C^{2}$ function $f: R \rightarrow R$ such that $f(0)=0$ and $f^{\prime}(0)=\alpha_{0}$. Then our desired function $\rho$ is given by

$$
\rho(Z)=\rho_{1}(Z)+f(\phi(Z)) .
$$

Our assertion 1 in Proposition 3.4 follows from the fact that

$$
\left.\frac{\partial \rho(Z)}{\partial Z_{v}^{u}}\right|_{Z=I_{n}}=\left.\frac{\partial \rho_{1}(Z)}{\partial Z_{v}^{u}}\right|_{Z=I_{n}}=C_{v}^{u}\left(I_{n}\right)+i D_{v}^{u}\left(I_{n}\right)
$$

and that our matrix $i e\left(I_{n}\right)$ is nonzero. For part 2, we note that if $Z \in \mathrm{U}(n)$ is near $I_{n}, \phi(Z)=\varphi_{t}^{s}(Z)=\psi_{t}^{s}(Z)=0$, and hence $\mathrm{U}(n) \subset$ $b \mathscr{D}$. For the inclusion that $\mathscr{U} \cap \widehat{\mathrm{U}(n)} \subset \mathscr{D}$, we note that for $Z \in \widehat{\mathrm{U}(n)}$ near $I_{n}$,

$$
\left(\phi_{t}^{s}(Z)+i \psi_{t}^{s}(Z)\right) \leq 0, \quad\left(C_{t}^{s}(Z)+i D_{t}^{s}(Z)\right) \geq 0 .
$$

Therefore, Lemma 3.1 and the fact that at $I_{n}, f \circ \phi$ vanishes to the second order while the function $\rho_{1}$ vanishes only to the first order, yield our result. For part 3, we note that the complex tangent space of $b \mathscr{D}$ at $P \in \Sigma$ is defined by

$$
\begin{aligned}
T_{P}^{C}(b \mathscr{D}) & =\left\{\Xi=\left(\xi_{v}^{u}\right)_{n \times n}:\left.\sum_{u, v=1}^{n} \xi_{v}^{u} \frac{\partial \rho(Z)}{\partial Z_{v}^{u}}\right|_{Z=P}=0\right\} \\
& =\left\{\Xi=\left(\xi_{v}^{u}\right)_{n \times n}:\left.\sum_{u, v=1}^{n} \xi_{v}^{u} \frac{\partial \rho_{1}(Z)}{\partial Z_{v}^{u}}\right|_{Z=P}=0\right\} .
\end{aligned}
$$

A computation shows that at $P, \Xi \in T_{P}^{C}(b \mathscr{D})$ if and only if the following equality holds

$$
\sum_{u, v, k=1}^{n} \xi_{v}^{u} \overline{P_{v}^{k}}\left(C_{k}^{u}(P)+i D_{k}^{u}(P)\right)=0 .
$$

Thus the inclusion $T_{P}(\Sigma) \subset T_{P}^{C}(B \mathscr{D})$ follows from the fact that the skew-hermitian matrix $e(P)$ is in $\left(T_{P}(\Sigma) \overline{P t}\right)^{\perp}$, i.e., $e(P)$ has to satisfy the equality $(*)$ for all $\Xi \in T_{P}(\Sigma)$.

To prove the last part, it is enough to show that the Hessian of $\rho(Z)$ is strictly positive definite when $P=I_{n}$. This is obtained from Lemma 3.2 and the following identity

$$
\left.\left(\frac{\partial^{2} \rho(Z)}{\partial Z_{k}^{u} \partial \overline{Z_{l}^{v}}}\right)\right|_{Z=I_{n}}=\left(\delta_{l}^{k}\left(C_{v}^{u}\left(I_{n}\right)+i D_{v}^{u}\left(I_{n}\right)\right)\right)_{n^{2} \times n^{2}}+B+\left.f^{\prime}(0) H(\phi)\right|_{Z=I_{n}} \text {. }
$$

Thus, we finish the proof of our proposition. 
The global version of Proposition 3.4 is stated in the following proposition that ensures that the domain $\mathscr{D}$ can be chosen globally as a strictly pseudoconvex domain. The proof is the same as that given by Saerens in [11] for polydiscs.

Proposition 3.5. Given a point $P \in \Sigma$, there exists a strictly pseudoconvex domain $\mathscr{D} \subset C^{n^{2}}$ with $C^{2}$ boundary and an open subset $\mathscr{U}$ containing $P$ such that conclusions (2) with (3) in Proposition 3.4 hold.

It is known $[5,10]$ that a $C^{1}$ submanifold $M$ in the boundary of a strictly pseudoconvex domain $D$ is a peak-interpolation set if and only if for each point $P \in M, T_{P}(M) \subset T_{P}^{C}(b D)$. Thus together with Proposition 3.5, we can assert, following the lines in Saerens [4], that $\Sigma$ is a local peak set and an interpolation set for $A(\widehat{\mathrm{U}(n)})$, provided that $\Sigma$ satisfies the open cone condition at every point. Since, for every point $P$ in $\mathrm{U}(n)$, the function

$$
f_{P}(Z)=\frac{\sum_{k, l=1}^{n} Z_{l}^{k} \overline{P_{l}^{k}}}{n}, \quad \text { for } Z \in \widehat{\mathrm{U}(n)},
$$

peaks only at the point $P, P$ is a peak point for $A(\widehat{\mathrm{U}(n)})$. Our sufficient condition in Theorem 2.3 is then an immediate consequence of a result, due to Varopoulos in [14], which implies that, for a bounded domain $D$, an interpolation set for $A(D)$ of which every point is also a peak point for $A(D)$ is actually a peak-interpolation set for $A(D)$.

Our proof of the necessity in Theorem 2.3 is based on the following two theorems (cf. [9]).

TheOREM (Nagel and Rudin). Let the segment $(0,1) \subset R$ be one edge of an open rectangle $Q$ in the upper half-plane of $C$. Suppose

(a) $f: Q \rightarrow C$ is a bounded $C^{1}$-function, and

(b) $\frac{\partial f}{\partial \bar{z}} \in L^{p}(Q)$ for some $p>1$.

Then $\lim f(x+i y)$ exists for almost all $x \in(0,1)$, as $y \rightarrow 0$.

TheOREM (Nagel and Rudin). If $\mathscr{D} \Subset C^{n}$ is a domain and if $K \subset$ $b \mathscr{D}$ is a peak set for $A(\mathscr{D})$, then there exists an $F \in H^{\infty}(\mathscr{D})$ which has no limit along any curve in $\mathscr{D}$ that ends at a point of $K$.

Nagel and Wainger obtained several more general results. For details, see [10]. 
Suppose now that $\sigma$ is a peak set for $A(\widehat{\mathrm{U}(n)})$ and that there is a point, say $P$, at which there is a non-zero vector $\xi \in T_{P}(\Sigma)$ such that $\xi \overline{P^{t}} \in \mathscr{P}_{+} \cup \mathscr{P}_{-}$. Choose a $C^{2}$ simple closed curve $\gamma$ in $\mathrm{U}(n)$ so that $P \in \gamma$ and $\gamma$ is the image of some $C^{2}$ periodic mapping $\varphi: R \rightarrow \mathrm{U}(n)$ with $\varphi(0)=P$ and $\varphi^{\prime}(0)=\xi$. Let $d=\operatorname{Max}\left\{\left\|\varphi^{\prime}\right\|_{\infty},\left\|\varphi^{\prime \prime}\right\|_{\infty}\right\}<\infty$. Assume that $i \varphi^{\prime}(0) \overline{\varphi^{t}(0)}<0$. (The same proof works for the case when the matrix $i \varphi^{\prime}(0) \overline{\varphi^{t}(0)}$ is positive definite.) Then this matrix remains negative definite for small $x$ in $R$. Without loss of generality, we assume that $\varphi(x+2)=\varphi(x)$. Choose a function $\psi:(-1,1) \rightarrow$ $C^{n^{2}}$ such that

$$
\int_{-1}^{1} \psi_{l}^{k}(x) d x=1, \quad \int_{-1}^{1}\left|x \psi_{l}^{k}(x)\right| d x<\frac{1}{d} .
$$

The existence of such a function is seen as follows. Let $c=\frac{1}{2 d}>0$, and let $\lambda$ be a function on $[0,1]$ with $\lambda(0)=\lambda^{\prime}(0)=\lambda^{\prime \prime}(0)=0$, $\lambda(1)=1 / 2, \lambda$ positive and increasing on $[0,1]$ such that $\int_{0}^{1} \lambda(x) d x$ $>1 / 2-c$. Then by extending $\lambda$ to $[-1,0]$ as an odd function, the function $\lambda^{\prime}(x)$ has the desired properties.

Define $u_{l}^{k}(x, y)=\int_{-1}^{1}\left(\varphi_{l}^{k}\right)^{\prime}(x+t y) \psi_{l}^{k}(t) d t$ and

$$
\Phi(x+i y)=\varphi(x)+i y u(x, y)
$$

for $x$ near 0 and $y>0$. Since our matrix $i \varphi^{\prime}(x) \overline{\varphi^{t}(x)}$ is negative definite when $x=0$, we can find a positive number $e$ so that for $x$ near 0 ,

$$
\operatorname{Im}\left(\sum_{l=1}^{n} \varphi_{l}^{\prime k}(x) \overline{\varphi_{l}^{k}(x)}\right)>e>0
$$

for $k=1, \ldots, n$. Therefore $\varphi$ and $\Phi$ have the following properties for $z=x+i y$ :

1. $\Phi(x)=\varphi(x)$ and $\left.\frac{\partial \Phi(z)}{\partial y}\right|_{y=0}=i \varphi^{\prime}(x)$.

2. There exist two small positive constants $\varepsilon, \eta$ such that under the mapping $\Phi$, the region $(-\varepsilon, \varepsilon) \times[0, \eta]$ is mapped into $\widehat{\mathrm{U}(n)}$ and

$$
\operatorname{Max}_{x \in[-\varepsilon, \varepsilon]}\left|\varphi^{\prime}(x)-\varphi^{\prime}(x+y)\right|<d y<e .
$$

3. $\left|u_{x}(x, y)\right| \leq\left\|\phi^{\prime}\right\|_{\infty},\left|u_{y}(x, y)\right| \leq 1$, and $\left|\frac{\partial \Phi(z)}{\partial \bar{z}}\right| \leq C<\infty$.

Let $\Phi(z)=\left(\Phi^{1}(z), \ldots, \Phi^{n}(z)\right)$, where the $\Phi^{k}$ 's are the columns 
of $\Phi$. Then for $1 \leq k \leq n$ and $z$ near 0

$$
\begin{aligned}
1-\left|\Phi^{k}(x+i y)\right|^{2}= & 1-\sum_{l=1}^{n}\left|\varphi_{l}^{k}(x)+i y u_{l}^{k}(x, y)\right|^{2} \\
= & 2 y\left(\operatorname{Im} \sum_{l=1}^{n}\left(\varphi_{l}^{k}\right)^{\prime}(x) \overline{\varphi_{l}^{k}(x)}\right) \\
& +2 y\left(\operatorname{Im} \sum_{l=1}^{n} \varphi_{l}^{k}(x)\left(u_{l}^{k}(x, y)-\left(\varphi_{l}^{k}\right)^{\prime}(x)\right)\right) \\
& -y^{2}\left|u_{l}^{k}(x, y)\right|^{2}>e y,
\end{aligned}
$$

since second and last term in this equality are $O\left(y^{2}\right)$ by the above property (2). Now $\mathrm{U}(n)$ is contained in the set $S, S=S^{2 n-1} \times \cdots \times$ $S^{2 n-1}$, the product of $n$ copies of $(2 n-1)$-sphere. The distance from the point $\Phi(z)$ to $\mathrm{U}(n)$ is greater than or equal to that to $S$. Suppose $\zeta$ to be a point in $S$. Then

$$
\begin{aligned}
\left|\Phi^{k}(z)-\zeta^{k}\right|^{2} & =1+\left|\Phi^{k}(z)\right|-2 \operatorname{Re}\left\langle\zeta^{k}, \Phi^{k}(z)\right\rangle \\
& \geq 1-\left|\Phi^{k}(z)\right|^{2}>e y .
\end{aligned}
$$

Therefore $\operatorname{dist}(\Phi(z), \mathrm{U}(n))>e y$. If $F$ is a function in $A(\widehat{\mathrm{U}(n)})$, by letting $f(z)=F(\Phi(z))$, we have following estimate for small $y$ :

$$
\left|\frac{\partial f(z)}{\partial \bar{z}}\right| \leq \frac{\|F\|_{\infty}}{\operatorname{dist}(\Phi(z), \mathrm{U}(n))}\left|\frac{\partial \Phi_{l}^{k}(z)}{\partial \bar{z}}\right|=O\left(y^{-1 / 2}\right) .
$$

Therefore $\frac{\partial f(z)}{\partial \bar{z}}$ belongs to $\mathscr{L}^{p}(Q)$ for some rectangle $Q$ in the upper half plane, where $1<p<2$. Our theorem then follows from the theorem of Nagel and Rudin.

The proof of the sufficiency of Theorem 2.4 could have been carried out exactly in the same fashion, if one notes that our constructions and all the theorems we quoted there still work in the $C^{\infty}$ case. But since we no longer have a $C^{\infty}$ version of Varopoulos's theorem, we can only reach the conclusion that $\Sigma$ is a local peak set and an interpolation set for $A^{\infty}(\widehat{\mathrm{U}(n)})$.

Proof of the necessity in Theorem 2.4: Let $f \in A^{k}(\widehat{\mathrm{U}(n)})$ be such that $\left.f\right|_{\Sigma}=1$ and $|f|_{\widehat{\mathrm{U}(n) \backslash \Sigma}}<1$. Without loss of generality, we can assume that $f \neq 0$ in $\widehat{\mathrm{U}(n)}$. Let $\log (f(Z))=u(Z)+i v(Z)$ and

$$
\mathscr{M}=\left\{Z \in C^{n^{2}}, v(Z)=0\right\} \cap \mathrm{U}(n) .
$$

Then $\mathscr{M}$ contains $\Sigma$. First we show that near every point $P_{0} \in \Sigma, \mathscr{M}$ is a manifold of dimension $n^{2}-1$. This amounts to showing that there 
exists $\eta \in T_{P_{0}}(\mathrm{U}(n))$ such that $\eta(v) \neq 0$. Consider the holomorphic mapping $\phi$ from the unit disc $U$ in $C$ to $\widehat{\mathrm{U}(n)} \backslash \mathrm{U}(n)$ defined by $\phi(\xi)=\xi P_{0}$. Then $\phi(1)=P_{0}, f(\phi(1))=1$ and $|f(\phi(\xi))|<1$ for $\xi \in U$. Therefore, Hopf's lemma and the Cauchy-Riemann equations give $d v\left(\phi\left(e^{i \theta}\right)\right) / d \theta \neq 0$. By taking $\eta=\left.\phi_{*} \frac{\partial}{\partial \theta}\right|_{1} \in T_{P_{0}}(\mathrm{U}(n))$, we have $\eta(v) \neq 0$. Thus $d\left(\left.v\right|_{\mathrm{U}(n)}\right)$ does not vanish at point $P_{0}$. Next we claim that $\mathscr{M}$ satisfies the closed cone condition at every point of $\Sigma$. If not, say at $P$, we have that the set $T_{P}(\mathscr{M}) \overline{P^{t}}$ is not contained in the set $\mathscr{S} \backslash\left(\mathscr{P}_{+} \cup \mathscr{P}_{-}\right)$. Then there exists an $n \times n$ matrix $\xi$ in $T_{P}(\mathscr{M})$ such that $i \xi \overline{P^{t}}>0$ or $i \xi \overline{P^{t}}<0$, say the former. Let $A=$ $i \overline{P^{t}} \xi$. Then $\xi+P \overline{\xi^{t}} P=0$ implies that $A=P^{-1}\left(i \xi \overline{P^{t}}\right) P>0$. For $|\zeta| \leq 1$, we define a matrix function $M(\zeta)=I_{n}-c(1-\zeta) A$, where $c=1 / \max _{1 \leq i \leq n} \lambda_{i}>0$ with $\lambda_{i}$ the positive eigenvalues of the matrix $A$. (If $A<0$, we take $c=-1 / \max _{1 \leq i \leq n}\left|\lambda_{i}\right|<0$.) The eigenvalues of $M(\zeta)$ are $\mu_{i}=1-c(1-\zeta) \lambda_{i}$ with

$$
\left|\mu_{i}\right|=1-c \lambda_{i}\left(\frac{2(1-\operatorname{Re} \zeta)}{|1-\zeta|^{2}}-c \lambda_{i}\right)<1,
$$

since $2(1-\operatorname{Re} \zeta)>(1-\operatorname{Re} \zeta)^{2}+(\operatorname{Im} \zeta)^{2}=|1-\zeta|^{2} \cdot M(1)=I_{n}$. Therefore the mapping $\zeta \rightarrow P M(\zeta)$ is $C^{\infty}$ from the unit disc to $\widehat{\mathrm{U}(n)}$. The function $f(P M(\zeta))$ takes its maximum modulus only at $\zeta=1$. Hopf's lemma yields $\left.\frac{\partial|f|}{\partial r}\right|_{\zeta=1}>0$. Thus the Cauchy-Riemann equations give $\left.\frac{\partial v}{\partial \theta}\right|_{\zeta=1}>0$. Now we write $M(\zeta)=\left(a_{t}^{k}(\zeta)\right)$ and $v=$ $v(P M(\zeta))=v\left(\sum_{k=1}^{n} P_{k}^{s} a_{t}^{k}(\zeta)\right)$. Then $\left.\frac{d}{d \zeta} a_{t}^{k}(\zeta)\right|_{\zeta=1}=i \sum_{l=1}^{n} \xi_{l}^{k} \overline{P_{l}^{t}}$ and

$$
\begin{aligned}
0<\left.\frac{\partial v}{\partial \theta}\right|_{\zeta=1} & =\left.\left.\sum_{s, t=1}^{n} \frac{\partial v}{\partial z_{t}^{s}}\right|_{Z=P} \frac{\partial}{\partial \theta}\left(\sum_{k=1}^{n} P_{k}^{s} a_{t}^{k}(\zeta)\right)\right|_{\zeta=1} \\
& =\left.\sum_{s, t=1}^{n} \frac{\partial v}{\partial z_{t}^{s}}\right|_{Z=P} \sum_{k=1}^{n} i P_{k}^{s} \frac{d}{d \zeta} a_{t}^{k}(1) \\
& =-\left.\sum_{s, t=1}^{n} \frac{\partial v}{\partial z_{t}^{s}}\right|_{Z=P} \xi_{t}^{s},
\end{aligned}
$$

which contradicts the equality $\operatorname{Re}\left(\left.\sum_{s, t=1}^{n} \frac{\partial v}{\partial z_{t}^{s}}\right|_{Z=P} \xi_{t}^{s}\right)=0$, since $\xi \in$ $T_{P}(\mathscr{M})$. Thus we finish our proof of Theorem 2.4.

\section{Remarks.}

REMARK 4.1. Recall that $\mathrm{U}(n)$ is the image of $\mathscr{S}$, the set of all $n \times$ $n$ skew-Hermitian matrices, under the exponential map. Therefore, 
if $\Omega$ is an open subset in $R^{m}$ and if $\Psi$ is a $C^{k}$ mapping from $\Omega$ to $\mathscr{S}, \exp \circ \Psi$ is also a $C^{k}$ mapping with image in $\mathrm{U}(n)$. Under this correspondence, if the set $\exp (\Psi(\Omega))$ is a submanifold in $\mathrm{U}(n)$, the open cone condition and closed cone condition on $\exp (\Psi(\Omega))$ are converted to the following form:

open cone condition at point $P=\Psi(X)$ :

$$
\left\{\left.(J \Psi)\right|_{X} V: V \in R^{m}\right\} \subset \mathscr{S} \backslash\left(\overline{\mathscr{P}_{+} \cup \mathscr{P}_{-}}\right),
$$

closed cone condition at point $P=\Psi(X)$ :

$$
\left\{\left.(J \Psi)\right|_{X} V: V \in R^{m}\right\} \subset \mathscr{S} \backslash\left(\mathscr{P}_{+} \cup \mathscr{P}_{-}\right),
$$

where $J \Psi$ is the Jacobian of the mapping $\Psi$. Thus we can restate all theorems in $\S 2$ by imposing these revised cone conditions on $\Psi(X)$.

The proof of the equivalence of these two types of cone conditions is based on the following lemma:

LEMMA 4.1. Let $H=H\left(t_{1}, \ldots, t_{m}\right)$ be an $n \times n$ matrix that depends on the parameters $t_{1}, \ldots, t_{m}$. Then for any constant $s$

$$
\frac{\partial}{\partial t_{i}}\left(e^{s H}\right)=-\int_{0}^{s} e^{-\left(s-s_{1}\right) H} \frac{\partial H}{\partial t_{i}} e^{-s_{1} H} d s_{1} .
$$

Proof. Let $X(s, t)=\frac{\partial}{\partial t_{j}} e^{-s H}$. Then

$$
\begin{aligned}
\frac{d X}{d s} & =\frac{\partial}{\partial t_{j}} \frac{\partial}{\partial s} e^{-s H}=\frac{\partial}{\partial t_{j}}\left(-H e^{-s H}\right) \\
& =-H X-\frac{\partial H}{\partial t_{j}} e^{-s H}
\end{aligned}
$$

Hence $X(s, t)$ satisfies the inhomogeneous system

$$
\frac{d Y}{d s}=A Y(s)+B(s), \quad Y(0)=0
$$

which has the solution $Y(s)=\int_{0}^{s} e^{(s-\tau) A} B(\tau) d \tau$. As a consequence, we have the desired equality in the lemma.

By using this lemma, our equivalence follows immediately. For if we write $\Psi(X)=\left(\psi_{t}^{S}(X)\right)$ and $e^{\Psi(X)}=\left(e_{t}^{S}(X)\right)$, then for every 
$V=\left(v_{1}, \ldots, v_{m}\right) \in R^{m}$ we have

$$
\begin{gathered}
i e^{-\Psi(X)}\left(\sum_{l=1}^{m} \frac{\partial e_{t}^{s}(X)}{\partial x_{l}}\right)_{n \times n}=i e^{-\Psi(X)}\left(\sum_{l=1}^{m}\left(\frac{\partial e_{t}^{s}(X)}{\partial x_{l}}\right)_{n \times n} v_{l}\right) \\
=i e^{-\Psi(X)} \sum_{l=1}^{m} e^{\Psi(X)}\left(\int_{0}^{1} e^{-\tau \Psi(X)} \frac{\partial \Psi(X)}{\partial x_{l}} e^{\tau \Psi(X)} d \tau\right)_{n \times n} v_{l} \\
=\int_{0}^{1} e^{-\tau \Psi(X)}\left(i \sum_{l=1}^{m} \frac{\partial \psi_{t}^{s}(X)}{\partial x_{l}} v_{l}\right)_{n \times n} e^{\tau \Psi(X)} d \tau .
\end{gathered}
$$

As two conjugate matrices, the left-hand side is positive (or negative) definite if and only if the right-hand side is. This gives the equivalence of the two type of cone conditions.

There is an interesting consequence of the above equivalence. If we canonically embed the polydisc $T^{n}$ into $\mathrm{U}(n)$ as

$$
\left(z_{1}, \ldots, z_{n}\right) \in T^{n} \rightarrow \operatorname{diag}\left(z_{1}, \ldots, z_{n}\right) \in \mathrm{U}(n),
$$

then every submanifold $\Sigma$ in $T^{n}$ can be considered as a submanifold $\widetilde{\Sigma}$ in $\mathrm{U}(n)$ of the same smoothness. It follows that $\widetilde{\Sigma}$ satisfies the open (closed, resp.) cone condition in our definition if and only if $\Sigma$ satisfies the open (closed, resp.) cone condition in Burns and Stout [2] and Saerens [11]. Namely, if $\Omega$ is an open subset in $R^{m}$ and if $\Phi: R^{m} \rightarrow R^{n}$ is a nonsingular map of class $C^{2}$, then the open cone condition in [2] and [11]

$$
\left\{\Phi^{\prime}(X) V: V \in R^{m}\right\} \cap \overline{R_{+}^{n}}=\{0\},
$$

which implies that the set $\left\{\left(e^{i \Phi_{1}(X)}, \ldots, e^{i \Phi_{n}(X)}\right): X \in \Omega\right\}$ is a peakinterpolation set for the polydisc algebra $A\left(\mathrm{U}^{n}\right)$, holds if and only if

$$
\left\{J \Psi(X) V: V \in R^{m}\right\} \subset \mathscr{S} \backslash \overline{\mathscr{P}_{+} \cup \mathscr{P}_{-}},
$$

where $\Psi(X)=\operatorname{diag}\left(i \Phi_{1}(X), \ldots, i \Phi_{n}(X)\right) \in \mathscr{S}$. Moreover, the point $\left(e^{i \Phi_{1}(X)}, \ldots, e^{i \Phi_{n}(X)}\right)$ in $\mathrm{U}^{n}$ corresponds to the point

$$
\operatorname{diag}\left(e^{i \Phi_{1}(X)}, \ldots, e^{i \Phi_{n}(X)}\right)=\exp (\Psi(X)) \in \mathrm{U}(n) .
$$

Similarly, the closed cone condition in [2] and [11] can be obtained by the same argument. Therefore all parallel results in [2] and [11] about peak sets, interpolation sets and peak-interpolation sets for $A\left(T^{n}\right)$, $A^{\infty}\left(T^{n}\right)$ and $A^{\omega}\left(T^{n}\right)$ can be obtained as corollaries of our Theorem $2.1,2.2,2.3$ and 2.4 . 
REMARK 4.2. If a submanifold $\Sigma$ in $\mathrm{U}(n)$ happens to be a closed subgroup (Lie subgroup), e.g., $\mathrm{SU}(n)$, then the cone condition holds at every point if and only if it holds at one point, because we have following proposition.

Proposition 4.2. If $\Sigma$ is a subgroup of $\mathrm{U}(n)$ and satisfies the open cone condition at point $P_{0}$, then $\Sigma$ has the same property at every point $P$.

Proof. From the definition of the real space of $\mathrm{U}(n)$, we can derive that for any unitary matrix $U$,

$$
U T_{P_{0}}(\mathrm{U}(n))=T_{U P_{0}}(\mathrm{U}(n)) \text { and } T_{P_{0}}(\mathrm{U}(n)) U=T_{P_{0} U}(\mathrm{U}(n)) \text {. }
$$

Since $\Sigma$ is a subgroup of $\mathrm{U}(n), P P_{0}^{-1}$ also lies in $\Sigma$. Therefore $T_{P}(\mathrm{U}(n))=P P_{0}^{-1} T_{P_{0}}(\mathrm{U}(n))$. Let $\Sigma=\Psi(\Omega)$ with $\Psi$ defined in Remark 4.1. Assume $P=\Psi(X)$ and $P_{0}=\Psi\left(X_{0}\right)$. Then there exist $m^{2}$ functions $A\left(X, X_{0}\right)=\left(a_{k}^{j}\left(X, X_{0}\right)\right)_{m \times m}$ such that

$$
\frac{\partial \Psi(X)}{\partial x_{k}}=\Psi(X) \Psi\left(X_{0}\right)^{-1}\left(\left.\sum_{j=1}^{m} \frac{\partial \Psi(X)}{\partial x_{j}}\right|_{X=X_{0}} a_{k}^{j}\left(X, X_{0}\right)\right),
$$

since $\Sigma \subset \mathrm{U}(n)$ implies $T_{P}(\Sigma) \subset T_{P}(\mathrm{U}(n))$. We only need to show that the matrix $A\left(X, X_{0}\right)$ is nonsingular. But this is obvious, since we have

$$
\frac{\partial \Psi(X)}{\partial x_{k}}=\sum_{j, l=1}^{m} \frac{\partial \Psi(X)}{\partial x_{l}}(X) a_{j}^{l}\left(X_{0}, X\right) a_{k}^{j}\left(X, X_{0}\right),
$$

and this implies that $A\left(X, X_{0}\right) A\left(X_{0}, X\right)=I_{n}$.

REMARK 4.3. Let $\mathscr{D}=\mathscr{D}_{1} \times \cdots \times \mathscr{D}_{r}$ be a domain in $C^{N}$, where each $\mathscr{D}_{\nu}$ is a strictly pseudoconvex domain of $C^{n_{\nu}}$ with $C^{\infty}$ boundary. Let $\rho_{\nu}$ be a defining function for $\mathscr{D}_{\nu}$ and set $\Gamma=b \mathscr{D}_{1} \times \cdots \times b \mathscr{D}_{r}$ and $\tau_{P}^{\nu}=i \alpha_{\nu}(P)^{-1} \chi_{P}^{\nu}$ with $\chi_{P}^{\nu}=\left(\operatorname{grad} \rho_{\nu}\right)_{P}$ and $\alpha_{\nu}(P)=\left|\chi_{P}^{\nu}\right|, \nu=$ $1, \ldots, r$. For each vector $\lambda=\left(\lambda_{1}, \ldots, \lambda_{r}\right)$ with $\lambda_{\nu}>0$, we set

$$
\Lambda_{P}(\lambda)=\left\{\sum_{\nu=1}^{r} t_{\nu} \tau_{P}^{\nu}, t_{\nu} \in R \text { and } \sum_{\nu=1}^{r} t_{\nu} \lambda_{\mu}=0\right\} \text {. }
$$

T. Jimbo proved in [7] the following result: Let $M \subset \Gamma$ be a real $C^{\infty}$ submanifold. If $M$ is locally a peak set for $A^{\infty}(\mathscr{D})$, then there exists a vector-valued $C^{\infty}$ function $\lambda=\left(\lambda_{1}, \ldots, \lambda_{r}\right)$ with $\lambda_{\nu}>0$ such that

$$
T_{P}(M) \subset T_{P}^{C}(\Gamma) \oplus \Lambda_{P}(\lambda(P)) .
$$


Conversely, if $M$ satisfies the above inclusion, then it is locally a peak set and locally an interpolation set for $A^{\infty}(\mathscr{D})$.

In our case, as the unitary group $\mathrm{U}(n)$ is contained in the product of $n$-copies of unit sphere, we need consider the domain that is the product of $n$-copies of unit ball.

Proposition 4.3. Let $\Omega$ be an open set in $R^{m}$, and let $\Phi$ be a nonsingular $C^{\infty}$ mapping from $\Omega$ to $b B_{n} \times \cdots \times b B_{n}=S^{2 n-1} \times \cdots \times$ $S^{2 n-1}$. Set $\Sigma=\Phi(\Omega)$. If $\Sigma$ is a local peak set for $A^{\infty}(\widehat{\mathrm{U}(n)})$ may not be a local peak set for $A^{\infty}(\mathscr{D})$, where $\mathscr{D}=B_{n} \times \cdots \times B_{n}$, then at every point $P \in \Sigma, P=\Phi(X)$,

$$
\sum_{k=1}^{n} \lambda_{k}\left(\sum_{l=1}^{n} \overline{\Phi_{l}^{k}(X)} \frac{\partial \Phi_{l}^{k}(X)}{\partial x_{j}}\right)=0 \quad \text { for } 1 \leq j \leq m
$$

for some $\lambda=\left(\lambda_{1}, \ldots, \lambda_{n}\right)$ with $\lambda_{\nu}>0$.

Two comments about Proposition 4.3 are in order:

1. The condition in the proposition is also sufficient, but we only need the necessity so that we can compare the equality in Proposition 4.3 with our cone conditions.

2. The above equality implies that for any vector $V=\left(v_{1}, \ldots, v_{m}\right)$ the matrix $\sum_{j=1}^{m}\left(\overline{\Phi^{t}(X)} \frac{\partial \Phi(X)}{\partial x}\right) v_{j}$ is neither positive definite nor negative definite. Therefore if $\Sigma$ is contained in $\mathrm{U}(n)$, then it has to satisfy the closed cone condition. But a local peak set for $A^{\infty}(\widehat{\mathrm{U}(n)})$ may not be a local peak set for $A^{\infty}(\mathscr{D})$, as the following example shows.

EXAMPLE 4.4. Let $\Sigma$ be a smooth curve in $\mathrm{U}(2) \subset S^{3} \times S^{3}$ defined by

$$
\Sigma=\left\{\left(\begin{array}{cc}
c & d e^{i \alpha(t)} \\
-d e^{i \beta(t)} & c e^{i \alpha(t)+i \beta(t)}
\end{array}\right): t \in R\right\}
$$

here $c, d$ are real constants satisfying $c^{2}+d^{2}=1$. Then we have

1. If $\alpha^{\prime}(t) \beta^{\prime}(t)<0$ for all $t$, then $\Sigma$ is a local peak set for $A^{\infty}(\widehat{\mathrm{U}(n)})$.

2. $\Sigma$ is a local peak set for $A^{\infty}\left(B_{2} \times B_{2}\right)$ only if $\alpha^{\prime}(t) \beta^{\prime}(t)<$ $-\left(c \beta^{\prime}(t)\right)^{2}<0$ for all $t$. 
Proof of Proposition 4.3. It is easy to get that

$$
\begin{aligned}
& \Lambda_{P}(\lambda)=\left\{i\left(t_{1} z_{1}^{1}, \ldots, t_{1} z_{n}^{1}, \ldots, t_{n} z_{1}^{n}, \ldots, t_{n} z_{n}^{n}\right)_{p}:\right. \\
& \left.t=\left(t_{1}, \ldots, t_{n}\right) \in R^{n}, \text { and } \sum_{j=1}^{n} t_{j} \lambda_{j}=0\right\} .
\end{aligned}
$$

For each $V \in R^{m}, \sum_{j=1}^{m} \frac{\partial \Phi(X)}{\partial x_{j}} v_{j} \in T_{P}(\Sigma)$ implies that $\sum_{j=1}^{m} \frac{\partial \Phi(X)}{\partial x_{j}} v_{j}$ - $\left(i_{k} \Phi_{j}^{k}(X)\right)_{1 \leq k, j \leq n}$ must lie in $T_{P}^{C}(\Gamma)$, where $\Gamma=S^{2 n-1} \times \cdots \times$ $S^{2 n-1}$. That means that

$$
\sum_{l=1}^{n}\left(\sum_{j=1}^{m} \frac{\partial \Phi_{l}^{k}(X)}{\partial x_{j}} v_{j}-i t_{k} \Phi_{l}^{k}(X)\right) \overline{\Phi_{l}^{k}(X)}=0,
$$

for $k=1, \ldots, n$. Since $V$ is arbitrary, our result follows from the equality $\sum_{l=1}^{n}\left|\Phi_{l}^{k}(X)\right|^{2}=1$.

Acknowledgment. I would like to thank my advisor Professor Edgar Lee Stout for his great help. Without his steady, patient support and encouragement, it is impossible for me to finish this paper.

\section{REFERENCES}

[1] R. E. Bellman, Introduction to Matrix Analysis, McGraw-Hill, New York, 1970.

[2] D. Burns Jr. and E. L. Stout, Extending functions from submanifolds of the boundary, Duke Math. J., 43 (1976), 391-404.

[3] F. R. Harvey and R. O. Wells, Jr., Holomorphic approximation and hyperfunction theory on a $C^{1}$ totally real submanifold of a complex manifold, Math. Ann., 197 (1972), 287-318.

[4] M. Hakim and N. Sibony, Ensembles pics dans domaines strictement pseudoconvexes, Duke Math. J., 45 (1978), 601-607.

[5] G. M. Henkin and A. E. Tumanov, Interpolation submanifolds of pseudoconvex domains, Trans. Amer. Math. Soc., 115 (1980), 59-69.

[6] L. K. Hua, Harmonic analysis of functions of several complex variables in the classical domains, Transl. Math. Monographs, vol. 6, Amer. Math. Soc., Providence, RI, 1963.

[7] T. Jimbo and A. Sakai, Interpolation manifolds for products of strictly pseudoconvex domains, Complex Variables, 8 (1987), 222-341.

[8] J. M. Labonde, Thesis, Universite de Paris-Sud, Centre d'Orsay, 1985.

[9] A. Nagel and W. Rudin, Local behavior of bounded holomorphic functions, Canad. J. Math., 30 (1978), 583-592.

[10] A. Nagel and S. Wainger, Limit of bounded holomorphic functions along curves, Recent Developments in Several Complex Variables, Princeton University Press, Princeton, 1980, pp. 327-344.

[11] R. Saerens, Interpolation submanifolds, Ann. Sci. Norm. Sup. Pisa Cl. Sci. IV Ser., 11 (1984), 177-211. 
[12] - Interpolation Theory in $C^{n}:$ A survey, Lecture Notes in Math., vol. 1268, Springer-Verlag, 1986, pp. 158-188.

[13] E. L. Stout, Interpolation manifolds, Recent Developments in Several Complex Variables, Princeton University Press, Princeton, 1980, pp. 373-391.

[14] N. T. Varopoulos, Ensembles pics et ensembles d'interpolation pour les algebres uniformes, C. R. Acad. Sci. Paris Ser. A, 272 (1971), 866-867.

Received April 3, 1992 and in revised form October 23, 1992.

UNIVERSITY OF WASHINGTON

SEATtLE, WA 98195

Current address: Department of Mathematics

Temple University

Philadelphia, PA 19122 



\section{PACIFIC JOURNAL OF MATHEMATICS}

Founded by

E. F. BECKENBACH (1906-1982) F. Wolf (1904-1989)

\section{EDITORS}

Sun-Yung A. Chang

(Managing Editor)

University of California

Los Angeles, CA 90024-1555

chang@math.ucla.edu

\section{F. Michael Christ}

University of California

Los Angeles, CA 90024-1555

christ@math.ucla.edu

Herbert Clemens

University of Utah

Salt Lake City, UT 84112

clemens@math.utah.edu

\author{
ThOMAs ENRIGHT \\ University of California, San Diego \\ La Jolla, CA 92093 \\ tenright@ucsd.edu \\ Nicholas ERcolaNI \\ University of Arizona \\ Tucson, AZ 85721 \\ ercolani@math.arizona.edu \\ R. FINN \\ Stanford University \\ Stanford, CA 94305 \\ finn@gauss.stanford.edu \\ VAUGHAN F. R. Jones \\ University of California \\ Berkeley, CA 94720 \\ vfr@math.berkeley.edu
}

\author{
STEVEn KerCKHOFF \\ Stanford University \\ Stanford, CA 94305 \\ spk@gauss.stanford.edu
}

Martin ScharlemanN

University of California

Santa Barbara, CA 93106

mgscharl@math.ucsb.edu

\author{
Harold Stark \\ University of California, San Diego \\ La Jolla, CA 92093 \\ V. S. VARADARAJAN \\ University of California \\ Los Angeles, CA 90024-1555 \\ vsv@math.ucla.edu
}

\section{SUPPORTING INSTITUTIONS}

UNIVERSITY OF ARIZONA

UNIVERSITY OF BRITISH COLUMBIA

CALIFORNIA INSTITUTE OF TECHNOLOGY

UNIVERSITY OF CALIFORNIA

UNIVERSITY OF MONTANA

UNIVERSITY OF NEVADA, RENO

NEW MEXICO STATE UNIVERSITY

OREGON STATE UNIVERSITY

\author{
UNIVERSITY OF OREGON \\ UNIVERSITY OF SOUTHERN CALIFORNIA \\ STANFORD UNIVERSITY \\ UNIVERSITY OF HAWAII \\ UNIVERSITY OF UTAH \\ WASHINGTON STATE UNIVERSITY \\ UNIVERSITY OF WASHINGTON
}

The Supporting Institutions listed above contribute to the cost of publication of this Journal, but they are not owners or publishers and have no responsibility for its content or policies.

Mathematical papers intended for publication in the Pacific Journal of Mathematics should be in typed form or offset-reproduced (not dittoed), double spaced with large margins. Please do not use built up fractions in the text of the manuscript. However, you may use them in the displayed equations. Underline Greek letters in red, German in green, and script in blue. The first paragraph must be capable of being used separately as a synopsis of the entire paper. In particular it should contain no bibliographic references. Please propose a heading for the odd numbered pages of less than 35 characters. Manuscripts, in triplicate, may be sent to any one of the editors. Please classify according to the 1991 Mathematics Subject Classification scheme which can be found in the December index volumes of Mathematical Reviews. Supply name and address of author to whom proofs should be sent. All other communications should be addressed to the managing editor, or Julie Honig, University of California, Los Angeles, California 90024-1555.

There are page-charges associated with articles appearing in the Pacific Journal of Mathematics. These charges are expected to be paid by the author's University, Government Agency or Company. If the author or authors do not have access to such Institutional support these charges are waived. Single authors will receive 75 free reprints; joint authors will receive a total of 100 free reprints. Additional copies may be obtained at cost in multiples of 50 .

The Pacific Journal of Mathematics (ISSN 0030-8730) is published monthly except for July and August. Regular subscription rate: $\$ 215.00$ a year (10 issues). Special rate: $\$ 108.00$ a year to individual members of supporting institutions.

Subscriptions, orders for numbers issued in the last three calendar years, and changes of address should be sent to Pacific Journal of Mathematics, P.O. Box 4163, Berkeley, CA 94704-0163, U.S.A. Old back numbers obtainable from Kraus Periodicals Co., Route 100, Millwood, NY 10546.

The Pacific Journal of Mathematics at University of California, c/o Department of Mathematics, 981 Evans Hall, Berkeley, CA 94720 (ISSN 0030-8730) is published monthly except for July and August. Second-class postage paid at Berkeley, CA 94704, and additional mailing offices. POSTMASTER: send address changes to Pacific Journal of Mathematics, P.O. Box 4163, Berkeley, CA 94704-0163.

PUBLISHED BY PACIFIC JOURNAL OF MATHEMATICS at University of California, Berkeley, CA 94720, A NON-PROFIT CORPORATION

This publication was typeset using $\mathcal{A} \mathcal{M} \mathcal{S}-\mathrm{T}_{\mathrm{E}} \mathrm{X}$,

the American Mathematical Society's $T_{E} X$ macro system.

Copyright (c) 1994 by Pacific Journal of Mathematics 


\section{PACIFIC JOURNAL OF MATHEMATICS}

Volume $165 \quad$ No. $1 \quad$ September 1994

The effect of dimension on certain geometric problems of irregularities of 1 distribution

RALPH ALEXANDER

The structure of $\mathrm{sl}(2,1)$-supersymmetry: irreducible representations and primitive ideals

Didier ARnAL, HÉdi BENAMOR and GEORges PinCZON

Periods and Lefschetz zeta functions

Josefina CASASAYas, JaUme Llibre and ANA Nunes

On closed hypersurfaces of constant scalar curvatures and mean curvatures in 67 $S^{n+1}$

\section{SHAOPING CHANG}

$R$-groups and elliptic representations for $\mathrm{SL}_{n}$

DAVID GOLDBERG

The boundary distortion of a quasiconformal mapping

JuHA HeINONEN and PEKKA KosKela

Strongly approximately transitive group actions, the Choquet-Deny theorem, 115 and polynomial growth

\section{WOJCIECH JAWORSKI}

$q$-canonical commutation relations and stability of the Cuntz algebra

PALle E. T. Jorgensen, L. M. Schmitt and Reinhard Frank

WERNER

Complete open manifolds of non-negative radial curvature

YOSHIROH MACHIGASHIRA

Perturbations of certain reflexive algebras

DAVID RYDER PITTS

Interpolation submanifolds of the unitary group 